\title{
Fractional Micro-ablative CO2 Laser as Therapy in Penile Lichen Sclerosus
}

\author{
Piero Campolmi ${ }^{1}$, Giovanni Cannarozzo ${ }^{2}$, Luigi Bennardo ${ }^{3 *}$, Alessandro Clementi ${ }^{2}$, Mario Sannino ${ }^{2}$, \\ Steven Paul Nisticò ${ }^{3}$
}

${ }^{1}$ Department of Dermatology, Università di Firenze, Florence, Italy

${ }^{2}$ Department of Dermatology, Tor Vergata University, Rome, Italy

${ }^{3}$ Department of Health Sciences, Unit of Dermatology, - Magna Graecia University, Catanzaro, Italy

\author{
*Correspondence to \\ Luigi Bennardo, \\ Department of Health Sciences, \\ Viale Europa SNC 88100 Magna \\ Graecia University Catanzaro \\ (CZ) Italy. \\ Tel: +3909613647195; \\ Email: luigibennardo10@gmail. \\ com
}

Received: September 11, 2020

Accepted: May 16, 2021

Published online October 18

2021

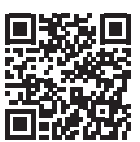

\section{Introduction}

Lichen sclerosus (LS) is a chronic inflammatory cutaneous disease interesting mainly the anogenital region. This disease affects patients of all ages, although it is rarely observed in infants. The disease usually appears in the fifth or sixth decade. ${ }^{1,2}$ Etiology is unknown, but the immune system seems to play a pathogenetic role in genetically predisposed patients. LS is a relapsing disease that can lead to atrophy, scarring, and functional impairment.

Genital LS is linked to an augmented risk of developing spinous cell carcinoma in the affected area. ${ }^{3}$ The diagnosis of this condition is mainly clinical, and biopsies should be performed only in selected cases. ${ }^{4}$

Penile LS mainly involves the prepuce and the glans. Foreskin becomes tightened and nonretractile with the progression of the disease. The involved areas show a significant reduction in elasticity, and the skin is prone to fissuring during sexual activities. ${ }^{3}$ LS increases the risk of complications such as infections, phimosis, and urethral stenosis. ${ }^{5}$

Chronic irritation, trauma, and occlusion under the prepuce represent precipitating factors. ${ }^{3}$ Topical steroids are the gold standard for treatment. In most cases, penile LS may be cured by circumcision, but this procedure is not well accepted by patients. ${ }^{4}$ Laser therapy is a further possible option for the treatment of LS. Researchers have reported using a fractional micro-ablative $\mathrm{CO} 2$ laser to treat genital LS. ${ }^{6-8}$ This technology may be an effective form of treatment for patients with penile LS.

\section{Case Presentation}

We report the case of a 69-year-old man diagnosed with penile LS. We observed some fissured sclerotic plaques on his tightened prepuce (Figures 1 and 2). The disease did not respond to different treatments with calcineurin inhibitors and topical steroids. The patient reported burning and sexual dysfunction due to the inelastic skin, and he refused circumcision.

Researchers carried out treatments using a fractional micro-ablative CO2 laser (SmartXide2, DEKA, M.E.L.A, Florence, Italy).

Parameters used were as follows: power, 10-13 W; spacing between dots, 500-600 $\mu \mathrm{m}$; number of pulses on the same spot (stack), 1-2; emission mode, high pulse (HP).

Researchers repeated the treatment until an excellent clinical and cosmetic result with a monthly interval. Five sessions were necessary for a good outcome. Researchers applied a topical anesthetic cream for 1 hour in occlusive dressing to relieve the pain before each treatment. The

Please cite this article as follows: Campolmi P, Cannarozzo G, Bennardo L, Clementi A, Sannino M, Nisticò SP. Fractional Micro-ablative CO2 Laser as Therapy in Penile Lichen Sclerosus. J Lasers Med Sci. 2021;12:e61. doi:10.34172/jlms.2021.61. 


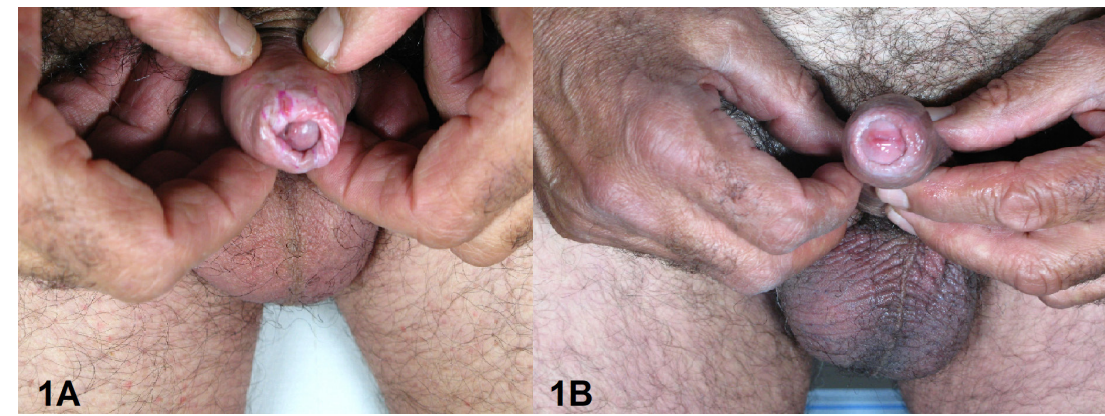

Figure 1. Penile Lichen Sclerosus Before (a) and After (b) Treatment.

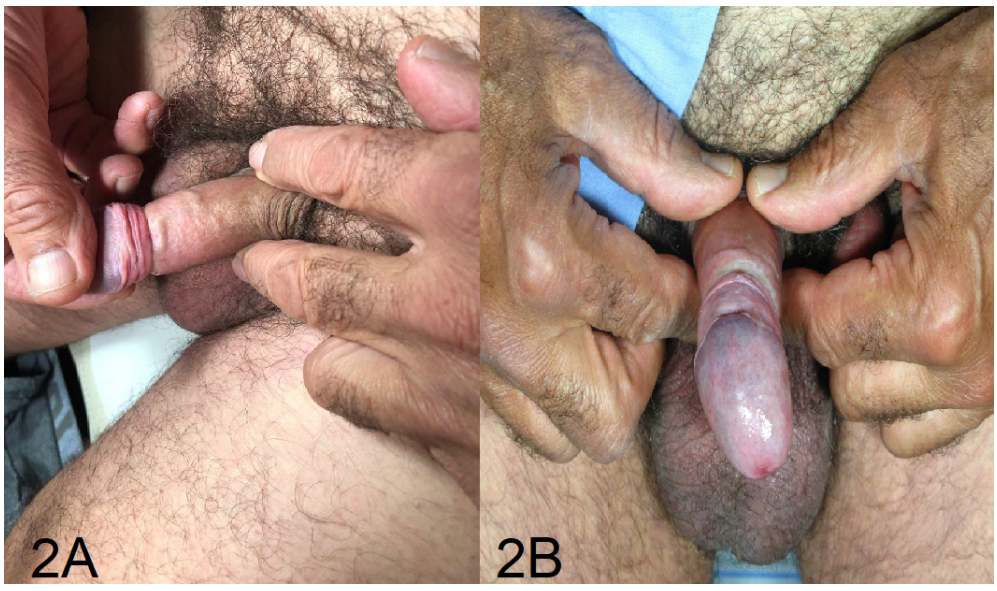

Figure 2. Penile Lichen Sclerosus Before (a) and After (b) Treatment.

patient tolerated the treatment well, and he did not report any side effects.

At the end of the procedure, an antibacterial ointment was applied to the treated surfaces twice a day for two weeks to favor reepithelization.

Results were evaluated with digital pictures taken at baseline and 14 weeks after the last treatment using a digital camera system, Anthology (DEKA M.E.L.A., Florence, Italy).

After the first treatment session, a significant improvement of symptomatology and increased skin elasticity were noted.

Pain during the treatment was minimal or absent. No other side effects were reported. The patient had an improvement as he did not feel any burning sensation and he was able to resume regular sexual activity. The replacement of sclerotic-fissured mucous tissue with a healthy one and the complete resolution of lichenification were observed after the last treatment. The clinical outcome showed no relapse of disease after 14 weeks (Figures 1 and 2).

\section{Discussion}

LS is a complex inflammatory disease. Standard medical therapy includes emollients, potent topical corticosteroids, and topical calcineurin inhibitors. ${ }^{3}$ Other therapies such as hydroxyurea, retinoids, ciclosporin, methotrexate, and steroid injections may be used in selected treatmentresistant cases. ${ }^{4}$

Circumcision is curative in most cases, but patients often refuse this procedure.

Laser therapy has been described for LS with variable results. ${ }^{2,9}$

In this study, we report our experience with fractional micro-ablative $\mathrm{CO} 2$ laser to treat penile LS.

This laser produces thermal columns heating and denaturing collagen with subsequent neo-collagenogenesis (biostimulation) in the treated areas..$^{10}$ Skin irradiation also induces the activation of heat shock proteins. These proteins modulate the secretion of different cytokines. The final effect is a restoration of tissue differentiation and neoangiogenesis. ${ }^{11,12}$

Fractional $\mathrm{CO} 2$ treatment is a safe and painless procedure that can be applied to genitalia.

Several authors have used this technology to treat genital LS with positive results. ${ }^{6,7}$

We have decided to treat our patient with the fractional $\mathrm{CO} 2$ micro-ablative laser considering the opposite sex results.

Mercuri et al reported 2 cases of penile LS treated with a combination of a fractional $1927 \mathrm{~nm}$ thulium fiber laser and photodynamic therapy. Both patients received three 
monthly treatments. Results were then assessed three months after the last treatment and then after two years, reporting no disease relapse. ${ }^{9}$

In our case, five sessions have been necessary to obtain optimal cosmetic results; the higher number of treatments may be related to a worse clinical condition than the other cases of penile LS reported in the literature, rather than minor effectiveness of fractional CO2 laser. The fractional $\mathrm{CO} 2$ laser having a major affinity to water can generate more in-depth columns and ablate skin surface when compared to the $1927 \mathrm{~nm}$ thulium fiber non-ablative laser that has a minor affinity. ${ }^{13,14}$

We evaluated the patient 14 weeks after the last treatment, and no relapse was observed. Although no relapse was observed after the $1927 \mathrm{~nm}$ fractional thulium fiber laser, ${ }^{9}$ future treatment cycles may be necessary to maintain or improve the obtained results.

Various laser types have been proposed for the treatment of almost exclusively female LS. Fractional ablative lasers such as $\mathrm{CO} 2$ and erbium and fractional non-ablative lasers such as $1927 \mathrm{~nm}$ thulium and Nd: YAG laser therapy have been proposed in single case reports or small studies. ${ }^{13-15}$ No clinical trial has been performed in order to compare the proposed treatments.

Penile LS represents a therapeutic challenge because it is refractory to classical treatment. The risk of relapsing and complications must be considered.

After the first treatment session, the fractional microablative CO2 laser induced a marked improvement in our patient's quality of life. The effects of the treatment led to the recovery of a normal mucosal surface and function. The long-term efficacy of this treatment should be evaluated with several follow-ups.

In conclusion, the fractional micro-ablative $\mathrm{CO} 2$ laser represents a valid alternative in the treatment of penile LS, but further studies on a larger number of patients must confirm these results.

\section{Informed Consent}

Written informed consent was obtained from the patient.

\section{Conflict of Interests}

The authors declare that they have no conflict of interest.

\section{Funding}

None.

\section{Acknowledgment}

We want to thank the patient for his consent to anonymous publication of the results of this case.

\section{References}

1. Powell JJ, Wojnarowska F. Lichen sclerosus. Lancet.
1999;353(9166):1777-1783. doi:10.1016/s0140-6736(98)08228-2

2. Peterson CM, Lane JE, Ratz JL. Successful carbon dioxide laser therapy for refractory anogenital lichen sclerosus. Dermatol Surg. 2004;30(8):1148-1151. doi:10.1111/j.1524-4725.2004. 30343.x

3. Fistarol SK, Itin PH. Diagnosis and treatment of lichen sclerosus: an update. Am J Clin Dermatol. 2013;14(1):2747. doi:10.1007/s40257-012-0006-4

4. Kirtschig G, Becker K, Günthert A, Jasaitiene D, Cooper S, Chi CC, et al. Evidence-based (S3) Guideline on (anogenital) Lichen sclerosus. J Eur Acad Dermatol Venereol. 2015;29(10): e1-e43. doi:10.1111/jdv.13136

5. Kantere D, Löwhagen GB, Alvengren G, Månesköld A, Gillstedt M, Tunbäck P. The clinical spectrum of lichen sclerosus in male patients - a retrospective study. Acta Derm Venereol. 2014;94(5):542-546. doi:10.2340/00015555-1797

6. Baggish M.S.Fractional CO2 Laser Treatment for Vaginal Atrophy and Vulvar Lichen Sclerosus. J Gynecol Surg. 2016.309-317. doi:10.1089/gyn.2016.0099

7. Lee A, Lim A, Fischer G. Fractional carbon dioxide laser in recalcitrant vulval lichen sclerosus. Australas J Dermatol. 2016;57(1):39-43. doi:10.1111/ajd.12305

8. Balchander D, Nyirjesy P. Fractionated CO2 Laser as Therapy in Recalcitrant Lichen Sclerosus. J Low Genit Tract Dis. 2020;24(2):225-228. doi:10.1097/ LGT.0000000000000512

9. Mercuri SR, Brianti P, Foti A, Bartolucci M, Dattola A, Nisticò SP. Penile Lichen Sclerosus Treated with $1927 \mathrm{~nm}$ Thulium Fiber Laser and Photodynamic Therapy: A New Possible Therapeutic Approach. Photomed Laser Surg. 2018;36(6):333-336. doi:10.1089/pho.2017.4386

10. Cannarozzo G, Nisticò SP, Nouri K, Sannino M. Surgical Ablative Lasers and Fractional Microablative Lasers (Far Infrared): Organ Tissue. In: Atlas of Lasers and Lights in Dermatology.2020. doi:10.1007/978-3-030-31232-9

11. Filippini M, Del Duca E, Negosanti F, Bonciani D, Negosanti L, Sannino M, et al. Fractional $\mathrm{CO}_{2}$ Laser: From Skin Rejuvenation to Vulvo-Vaginal Reshaping. Photomed Laser Surg. 2017;35(3):171-175. doi: 10.1089/pho.2016.4173.

12. Prignano F, Campolmi P, Bonan P, Ricceri F, Cannarozzo G, Troiano M, et al. Fractional CO2 laser: a novel therapeutic device upon photobiomodulation of tissue remodeling and cytokine pathway of tissue repair. Dermatol Ther. 2009 Nov;22 Suppl 1:S8-15. doi: 10.1111/j.15298019.2009.01265.x.

13. Lee A, Lim A, Fischer G. Fractional carbon dioxide laser in recalcitrant vulval lichen sclerosus. Australas J Dermatol. 2016;57(1):39-43. doi: 10.1111/ajd.12305.

14. Bizjak Ogrinc U, Senčar S, Luzar B, Lukanović A. Efficacy of Non-Ablative Laser Therapy for Lichen Sclerosus: A Randomized Controlled Trial. J Obstet Gynaecol Can. 2019 ;41(12):1717-1725. doi: 10.1016/j.jogc.2019.01.023.

15. Hobson JG, Ibrahim SF, Mercurio MG. Recalcitrant Vulvar Lichen Sclerosus Treated with Erbium YAG Laser. JAMA Dermatol. 2019;155(2):254-256. doi: 10.1001/ jamadermatol.2018.4461. 